\title{
La révision du chapitre TARMED sur la radiologie apporte des avantages aux patients
}

TARMED Suisse

Correspondance:

TARMED Suisse

Stephan Hänsenberger

Président

Spitalgasse 9

CH-3011 Berne

Tél. 0313100555

Fax 0313100557

info@tarmedsuisse.ch

www.tarmedsuisse.ch
Pour la première fois depuis l'introduction du tarif TARMED pour les traitements ambulatoires dans les cabinets médicaux et les hôpitaux, c'est un chapitre entier qui vient d'être révisé avec le domaine radiologie/procédés d'imagerie. Désormais, les examens d'imagerie effectués sur le patient et les frais administratifs seront facturés séparément. Par ailleurs, la complexité du tarif des examens d'imagerie s'en trouve nettement réduite.

La solution élaborée par les quatre partenaires de négociations $\mathrm{FMH}, \mathrm{H}+$, AA/AM/AI et santésuisse satisfait aux directives de l'Office fédéral de la santé publique (OFSP). Le chapitre TARMED Radiologie révisé stipule que l'ensemble des coûts indirects associés à chaque consultation de patient sera désormais indemnisé par un forfait séparé.

Parallèlement, les taux d'indemnisation pour les examens seront réduits. Après avoir réclamé pendant des années l'ajustement des salaires et des prix dans les procédés d'imagerie médicale (ultrasons, tomographie informatisée, résonance magnétique et radiographie), les hôpitaux voient aujourd'hui leur revendication satisfaite par la révision du chapitre 30, et mise en œuvre par la version TARMED 1.05. L'ajustement en rien n'a modifié la haute qualité de la radiologie, que ce soit à l'hôpital ou en cabinet médical.

Globalement, la révision du chapitre TARMED Radiologie simplifie la structure tarifaire des examens d'imagerie et soutient les prescriptions légales relatives à la manipulation des rayons X. Conformément aux directives de l'OFSP, les modifications apportées à l'indemnisation financière des fournisseurs de prestations observent le principe de la neutralité des coûts.

Les répercussions de la révision doivent être surveillées dans les hôpitaux, les instituts de radiologie et les cabinets médicaux, et pouvoir être corrigées si nécessaire. 